\title{
Report of the Korean Association of External Quality Assessment Service on Glucose Point-of- Care Testing (2018-2019)
}

\author{
Jinsook Lim ${ }^{\oplus}$ and Gye Cheol Kwon \\ Department of Laboratory Medicine, Chungnam National University College of Medicine, Deajeon, Korea
}

\author{
Corresponding author: \\ Gye Cheol Kwon \\ Department of Laboratory Medicine, \\ Chungnam National University College \\ of Medicine, 266 Munhwa-ro, Jung-gu, \\ Daejeon 35015, Korea \\ Tel +82-42-280-7799 \\ E-mail kckwon@cnu.ac.kr
}

In the 2018 and 2019 glucose analysis by point-of-care testing, an external quality assessment was performed in two sessions. Quality control (QC) materials available on the market were used in these programs, and three levels of concentration were used during each session. Based on the data and results of the test items reported by each institution, statistical analyses were conducted by the manufacturers and with a test analyzer. From the analysis results, each institution was provided with an overall report and an institution-tailored report. The average response rate was $98.8 \%$. After the number of instruments that could be registered by an institution increased to 30 in 2017, the number of participating institutions spiked to 3,808 during the second round in 2019. The coefficients of variation (CVs) differed among the manufacturers and among different instruments from the same manufacturer, and such differences were comparatively greater than the CV of glucose of the routine chemistry program. Due to the fact that the commutability of the QC materials used for this program is not secured between different instruments as well as between test strips with different lot numbers within the same instrument, it is deemed necessary to obtain lot numbers when inputting institution results.

(Lab Med Qual Assur 2021;43:43-55)

Key Words Laboratory proficiency testing, Quality assurance, Glucose, Glucometer, Point-of-care testing

\section{서론}

전 세계의 약 $7 \%$ 의 유병률을 가진 당뇨는 지속적으로 증가하 고 있고, 그 진단과 치료에 있어 혈당의 측정은 무엇보다도 중요 하다[1]. Glucometer는 현장검사장비 중 가장 널리 사용되고 있 는 장비이며[2], 최근에는 환자들의 혈당상태를 적절히 모니터 링하고 간편하게 측정하기 위해 원내 검사실이 있음에도 불구 하고 검사실 이외의 공간, 즉 병동, 응급실, 중환자실 그리고 외 래에서 다양한 목적으로 사용되고 있다[3]. 검사실의 모든 장비 에 대한 검사실 간의 비교를 통한 정확성에 대한 확인이 필요하
고 glucometer에도 외부정도관리가 필요하다는 인식이 확대됨 에 따라 대한임상검사정도관리협회는 2016년부터 glucose 현장 검사에 대한 외부정도관리를 시행하였으며, 2016년부터는 한 기 관당 최대 5개, 2017년부터는 30개까지 참여가 가능하도록 그 범위를 확대하였다. 저자들은 2018년도와 2019년도에 시행한 glucose 현장검사에 대한 신빙도조사사업의 내용과 그 결과를 보고하고자 한다. 


\section{재료 및 방법}

\section{1. 조사항목 및 신빙도조사용 정도관리물질}

2018년부터 2019년까지 glucose 현장검사는 연간 2회에 걸 쳐 glucose 단일 종목에 대해 회차당 3 개의 검체로 신빙도조 사가 시행되었다. Liquichek Meter Trax Control (Bio-Rad Laboratories, Hercules, CA, USA)을 정도관리물질로 사용하였 으며, 발송일정 및 사용된 물질명 및 lot 번호 그리고 참여기관의 수는 Table 1에 나타나 있다.

\section{2. 결과 판정 및 분석}

각 기관에서 입력한 결과를 바탕으로 제조사 및 검사장비에 따른 통계분석을 시행하였고, 전체에 해당하는 통계, 동일 제조 사에 따른 기준분류 및 동일 장비를 사용하는 세분류에 따른 통 계를 각각 제시하였다. 각 참여기관에는 개별기관의 평가자료 를 나타내는 기관별 보고서 및 전체 참여기관의 평가결과를 나 타낸 공통보고서를 제공하였다. 기관별 보고서에는 각 기관의 결과에 대한 전체, 동일한 제조사에 기반한 기준분류 그리고 동 일한 장비에 기반한 세분류에 따른 참여기관 수, 평균(mean), 표준편차(standard deviation), 변이계수(coefficient of variation), 중앙값(median), 최소값, 최대값 그리고 표준편 차지수(standard deviation index)를 기록하였다. 그리고 공 통보고서에는 참여한 전체기관, 기준분류 및 세분류에 대한 참
여기관의 수, 평균, 표준편차, 변이계수, 중앙값, 최소값, 최대 값을 제시하였다. 평균, 표준편차 그리고 변이계수의 경우 해당 분류별 분석에서 75 percentile 값(Q3)과 25 percentile 값 (Q1)의 차(Q3-Q1; interquartile range, IQR)의 1.5배를 초 과하여 Q1보다 낮거나 Q3보다 높은 결과값(<Q1-1.5 IQR or $>\mathrm{Q} 3+1.5 \mathrm{IQR}$ )을 이상치로 제거한 후 다시 분석한 값을 제시하 였다. 세분류를 기준으로 해당 분류에 속하는 기관 수가 10 개 미만인 경우에는 평균, 표준편차, 변이계수, 표준편차지수를 제 시하지 않았으며, 기관 수가 3 개 미만인 경우에는 중앙값도 제 시하지 않았다. 또한 세분류를 기준으로 해당 분류에 속하는 기 관 수가 1 개인 경우에는 공통보고서에 포함되지 않았다. 판정은 기준분류 혹은 세분류에서 표준편차지수가 3 미만인 경우 적합 (acceptable)하다고 하였다.

\section{결과 및 고찰}

2018년에서부터 2019년까지 glucose 현장검사에 대한 회신기 관 수, 회신율 그리고 평가장비 수는 Table 1에 각각 표시하였으 며, 각 검사항목별 참여기관 혹은 참여장비의 수는 Table 2에 나 타냈다. 참여기관 수는 2016년부터 2019년까지 꾸준히 상승하 였으며(Fig. 1), 2016년 1차 196개에 비해 2019년 2차에는 357 개 기관이 참여한 것으로 나타나 $82.1 \%$ 의 증가율을 나타냈다. 참 여기관 수가 늘어났음에도 불구하고, 여전히 2019년 일반화학검

Table 1. Sample shipment schedules and materials used for the external quality assessments of glucose point-of-care testing in the 2018 and 2019 trials

\begin{tabular}{|c|c|c|c|c|}
\hline Trial & Shipment schedule & Sample no. (lot no.) & $\begin{array}{l}\text { No. of } \\
\text { laboratories } \\
\text { invited }\end{array}$ & $\begin{array}{c}\text { Participating } \\
\text { laboratories }(\%)\end{array}$ \\
\hline \multirow[t]{3}{*}{ 1st, 2018} & 2018. 4. 23. & GGP-18-01 (92621) & 344 & $338(98.3)$ \\
\hline & & GGP-18-02 (92692) & & \\
\hline & & GGP-18-03 (92693) & & \\
\hline \multirow[t]{3}{*}{ 2nd, 2018} & 2018. 9. 10. & GGP-18-04 (92711) & 344 & $338(98.3)$ \\
\hline & & GGP-18-05 (92712) & & \\
\hline & & GGP-18-06 (92713) & & \\
\hline \multirow[t]{3}{*}{ 1st, 2019} & 2019. 4. 22. & GGP-19-01 (92730) & 359 & $356(99.2)$ \\
\hline & & GGP-19-02 (92730) & & \\
\hline & & GGP-19-03 (92730) & & \\
\hline \multirow[t]{3}{*}{ 2nd, 2019} & 2019. 10. 28. & GGP-19-04 (96762) & 359 & $357(99.2)$ \\
\hline & & GGP-19-05 (96763) & & \\
\hline & & GGP-19-06 (96761) & & \\
\hline
\end{tabular}


Table 2. List of manufacturers and instruments that participated in the external quality assessment program for glucose point-of-care testing

\begin{tabular}{|c|c|c|c|c|c|}
\hline \multirow{2}{*}{ Manufacturer } & \multirow{2}{*}{ Instrument } & \multicolumn{4}{|c|}{ Trial } \\
\hline & & 1st, 2018 & 2nd, 2018 & 1st, 2019 & 2nd, 2019 \\
\hline \multirow[t]{4}{*}{ Abbott } & Freestyle Optimum & 32 & 33 & 38 & 22 \\
\hline & Freestyle Optimum Neo & 22 & 22 & 33 & 31 \\
\hline & Freestyle Optimum Neo H & 7 & 7 & 8 & 8 \\
\hline & Freestyle Optimum Xceed & 49 & 24 & 19 & 19 \\
\hline \multirow[t]{4}{*}{ Allmedicus } & GlucoDr. (AGM-2100) & 1 & 1 & 1 & \\
\hline & GlucoDr. Auto (AGM-4000) & 4 & 4 & 10 & 10 \\
\hline & GlucoDr. Plus (AGM-3000/B) & 101 & 68 & 26 & 15 \\
\hline & Others & & & 30 & 41 \\
\hline \multirow[t]{2}{*}{ Arkray Inc. } & Glucocard 01 & 14 & 9 & 10 & 10 \\
\hline & Glucocard II & 2 & 2 & 1 & 1 \\
\hline \multirow[t]{3}{*}{ Ascensia } & Breeze 2 & 18 & & & \\
\hline & Contour plus & 38 & 43 & 58 & 62 \\
\hline & Contour TS & 19 & 23 & 14 & \\
\hline \multirow[t]{2}{*}{ GC Medis } & Green check & & & & 26 \\
\hline & Others & & & & 4 \\
\hline \multirow[t]{6}{*}{ Infopia } & GlucoLAB & 13 & 17 & 20 & \\
\hline & GlucoLAB Auto-coding & 7 & 20 & 23 & 22 \\
\hline & Gluneo Lite & 1 & & & \\
\hline & Gluneo Plus & 118 & 133 & 165 & 190 \\
\hline & Mirae 3.3G+ & 5 & 5 & 5 & 5 \\
\hline & Others & 3 & & & \\
\hline \multirow[t]{14}{*}{ i-SENS } & ACURA plus & & & 48 & 52 \\
\hline & BAROZen & 52 & 66 & 59 & 61 \\
\hline & BAROZen $\mathrm{H}$ & 147 & 114 & 93 & 59 \\
\hline & BAROzen $\mathrm{H}$ expert & 317 & 421 & 442 & 396 \\
\hline & BAROzen $\mathrm{H}$ expert plus & 81 & 83 & 203 & 321 \\
\hline & BAROZen H plus & 129 & 145 & 170 & 154 \\
\hline & BAROZen II & & & 10 & 14 \\
\hline & BAROZen plus & & & 67 & 134 \\
\hline & CareSens Dual & & & 10 & 10 \\
\hline & CareSens II & 15 & 11 & 11 & 1 \\
\hline & CareSens N & 80 & 83 & 61 & 60 \\
\hline & CareSens N Voice & 103 & 118 & 113 & 97 \\
\hline & CareSens PRO & 78 & 81 & 107 & 104 \\
\hline & Others & 69 & 50 & & \\
\hline
\end{tabular}

(Continued on next page) 
Table 2. Continued

\begin{tabular}{|c|c|c|c|c|c|}
\hline \multirow{2}{*}{ Manufacturer } & \multirow{2}{*}{ Instrument } & \multicolumn{4}{|c|}{ Trial } \\
\hline & & 1st, 2018 & $2 n d, 2018$ & 1st, 2019 & 2nd, 2019 \\
\hline \multirow[t]{3}{*}{ Philosys } & Gmate check & & & 46 & 46 \\
\hline & Gmate Origin & & & 28 & 26 \\
\hline & Gmate WHeeL & & & 11 & 11 \\
\hline \multirow[t]{3}{*}{ Roche } & Accu-Check Active & 198 & 178 & 183 & 153 \\
\hline & Accu-Check Inform II & 411 & 413 & 485 & 461 \\
\hline & Accu-Check Performa & 619 & 673 & 722 & 722 \\
\hline \multirow[t]{11}{*}{ SD Biosensor } & SD CHECK GOLD (Meter 01GM10) & 36 & 37 & 40 & 38 \\
\hline & SD CHECK GOLD (System 01GC12) & 7 & 6 & & \\
\hline & SD CodeFree (Meter 01GM11) & 117 & 87 & 123 & 134 \\
\hline & SD CodeFree (System 01GC112) & 8 & 10 & 8 & 8 \\
\hline & SD GlucoNavii & & & 30 & \\
\hline & SD GlucoNavii GDH (Meter 01GM30) & & & 14 & 49 \\
\hline & SD GlucoNavii GDH (System 01GC32) & & & 6 & 19 \\
\hline & SD GlucoNavii Link 0.3 (Meter 01GM12) & 40 & 40 & 41 & 41 \\
\hline & SD GlucoNavii Link 0.3 (System 01GC122) & 12 & 12 & & \\
\hline & SD GlucoNavii NFC (Meter 01GM40) & 78 & 82 & 75 & 65 \\
\hline & SD GlucoNavii NFC (System 01GC42) & 6 & 11 & & \\
\hline Wise Meditech & Wisecheck & & & 13 & 13 \\
\hline Others & Others & 98 & 130 & 108 & 74 \\
\hline Total & & 3,155 & 3,262 & 3,788 & 3,803 \\
\hline
\end{tabular}

The instruments used were from the following companies: Abbott (Abbott Park, IL, USA), Allmedicus (Anyang, Korea), Arkray Inc. (St. Edina, MN, USA), Ascensia (Newbury, UK), GC Medis (Cheonan, Korea), Infopia (Anyang, Korea), i-SENS (Seoul, Korea), Philosys (Gunsan, Korea), Roche (Indianapolis, IN, USA), SD Biosensor (Suwon, Korea), and Wise Meditech (Anyang, Korea).

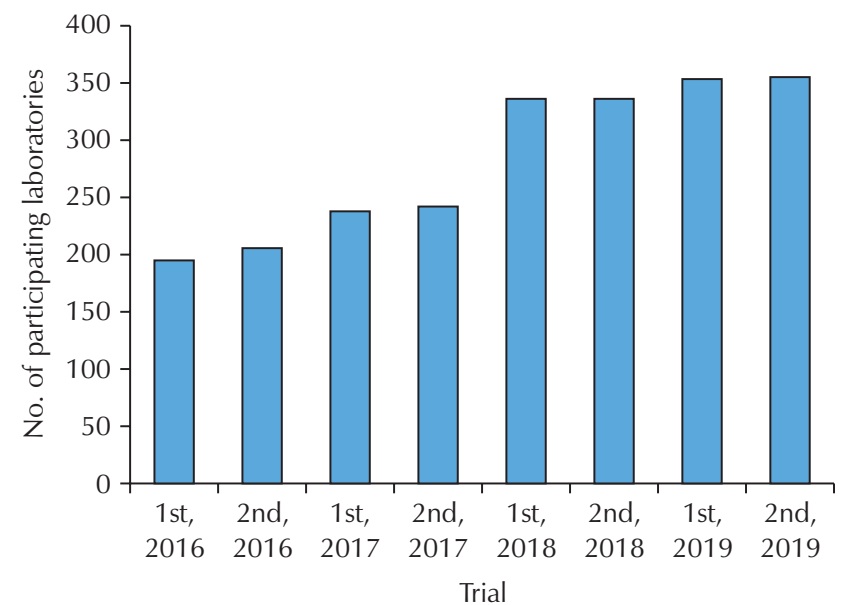

Fig. 1. The number of participating laboratories per each trial.
사의 glucose 항목 참여기관 수(1,664-1,719)에 비해 월등히 낮 아 glucose 현장검사에 대한 기관 참여율은 상대적으로 저조한 것으로 파악된다[4]. 참여기관 대부분은 검사실을 가지고 있는 병 원급으로 확인되었으며, 검사실 없이 glucometer가 사용되는 의 원급 기관(외래 혹은 요양병원 등)의 참여는 거의 없는 것으로 나 타났다. 현장검사의 경우 외부정도관리 프로그램에 참가한 횟수 가 많거나 혹은 그 기간이 길수록 검사의 질을 확보할 가능성이 높 아지는 것이 확인된 만큼 $[5,6]$, 현장검사장비에서의 외부정도관 리의 참여는 질 향상을 위한 필수적인 요소임이 강조되고 있다[7]. 또한 glucose 현장검사장비의 외부정관리에 대한 참여기관의 합 격률은 $90 \%$ 정도로 일반화학 검사항목인 glucose의 합격률이 98\%-99\%인 것과 비교할 때 낮은 것으로 보고되고 있으며[1], 외 부정도관리의 지속적인 참여를 통해 검사의 질을 궁극적으로 향상 시킬 수 있을 것으로 예상할 수 있다. 
Table 3. Distribution of instruments and mean, SD, and CVs (\%) for glucose point-of-care testing (GGP-18-01 to GGP-18-03)

\begin{tabular}{|c|c|c|c|c|c|c|c|c|}
\hline \multirow[b]{2}{*}{ Manufacturer } & \multirow[b]{2}{*}{ Instrument } & \multirow[b]{2}{*}{ No. } & \multicolumn{2}{|c|}{ GGP-18-01 } & \multicolumn{2}{|c|}{ GGP-18-02 } & \multicolumn{2}{|c|}{ GGP-18-03 } \\
\hline & & & Mean \pm SD & $\begin{array}{l}\text { CV } \\
(\%)\end{array}$ & Mean $\pm S D$ & $\begin{array}{c}\text { CV } \\
(\%)\end{array}$ & Mean $\pm S D$ & $\begin{array}{c}\text { CV } \\
(\%)\end{array}$ \\
\hline \multirow[t]{4}{*}{ Abbott } & Freestyle Optimum & 32 & $78.3 \pm 2.5$ & 3.2 & $143 \pm 6.9$ & 4.8 & $280.3 \pm 11.5$ & 4.1 \\
\hline & Freestyle Optimum Neo & 22 & $66.2 \pm 2.1$ & 3.1 & $152 \pm 6.1$ & 4.0 & $356.3 \pm 22.5$ & 6.3 \\
\hline & Freestyle Optimum Neo H & 7 & & & & & & \\
\hline & Freestyle Optimum Xceed & 49 & $78.5 \pm 4.2$ & 5.3 & $149.4 \pm 5.6$ & 3.7 & $293.2 \pm 17.3$ & 5.9 \\
\hline \multirow[t]{4}{*}{ Allmedicus } & GlucoDr. Auto (AGM-4000) & 4 & & & & & & \\
\hline & GlucoDr. Plus (AGM-3000/B) & 101 & $44.5 \pm 2.5$ & 5.7 & $105.7 \pm 4.4$ & 3.9 & $254.7 \pm 9.4$ & 3.7 \\
\hline & GlucoDr. (AGM-2100) & 1 & & & & & & \\
\hline & Others & & & & & & & \\
\hline \multirow[t]{2}{*}{ Arkray Inc. } & Glucocard 01 & 14 & $41.3 \pm 6.0$ & 14.5 & $97.9 \pm 12.9$ & 13.2 & $290.1 \pm 31.5$ & 10.9 \\
\hline & Glucocard II & 2 & & & & & & \\
\hline \multirow[t]{3}{*}{ Ascensia } & Breeze 2 & 18 & $39.7 \pm 0.8$ & 1.9 & $116.4 \pm 4.6$ & 4.0 & $331.7 \pm 8.8$ & 2.7 \\
\hline & Contour plus & 38 & $55.6 \pm 2.7$ & 4.8 & $111.2 \pm 3.8$ & 3.4 & $263.7 \pm 5.1$ & 1.9 \\
\hline & Contour T & 19 & $31.7 \pm 1.6$ & 4.9 & $94.9 \pm 6.6$ & 7.0 & $352.7 \pm 11.0$ & 3.1 \\
\hline \multirow[t]{2}{*}{ GC Medis } & Green check & & & & & & & \\
\hline & Others & & & & & & & \\
\hline \multirow[t]{6}{*}{ Infopia } & GlucoLAB & 13 & $53.0 \pm 0.0$ & 0.0 & $112.3 \pm 2.3$ & 2.1 & $286.3 \pm 5.2$ & 1.8 \\
\hline & GlucoLAB Auto-coding & 7 & & & & & & \\
\hline & Gluneo Lite & 1 & & & & & & \\
\hline & Gluneo Plus & 118 & $55.7 \pm 4.9$ & 8.8 & $88.1 \pm 5.3$ & 6.0 & $226.5 \pm 7.7$ & 3.4 \\
\hline & Mirae 3.3G+ & 5 & & & & & & \\
\hline & Others & 3 & & & & & & \\
\hline \multirow[t]{14}{*}{ i-SENS } & ACURA plus & & & & & & & \\
\hline & BAROZen & 52 & $38.1 \pm 3.1$ & 8.2 & $96.6 \pm 6.2$ & 6.4 & $263.6 \pm 35.7$ & 13.6 \\
\hline & BAROZen $\mathrm{H}$ & 147 & $41.0 \pm 7.1$ & 17.3 & $104.3 \pm 6.1$ & 5.8 & $304.1 \pm 23.1$ & 7.6 \\
\hline & BAROzen $\mathrm{H}$ expert & 317 & $52.4 \pm 3.7$ & 7.0 & $116.3 \pm 7.0$ & 6.0 & $330.5 \pm 14.5$ & 4.4 \\
\hline & BAROzen $\mathrm{H}$ expert plus & 81 & $53.0 \pm 1.8$ & 3.4 & $112.3 \pm 3.5$ & 3.1 & $327.9 \pm 9.1$ & 2.8 \\
\hline & BAROZen H plus & 129 & $53.3 \pm 2.5$ & 4.8 & $118.0 \pm 5.8$ & 4.9 & $339.1 \pm 16.1$ & 4.7 \\
\hline & BAROZen II & & & & & & & \\
\hline & BAROZen plus & & & & & & & \\
\hline & CareSens Dual & & & & & & & \\
\hline & CareSens II & 15 & $32.4 \pm 2.1$ & 6.6 & $96.1 \pm 6.3$ & 6.6 & $259.0 \pm 21.7$ & 8.4 \\
\hline & CareSens N & 80 & $39.0 \pm 3.2$ & 8.2 & $99.0 \pm 8.0$ & 8.1 & $276.6 \pm 45.9$ & 16.6 \\
\hline & CareSens N Voice & 103 & $41.0 \pm 4.2$ & 10.2 & $103.3 \pm 5.5$ & 5.3 & $291.9 \pm 17.7$ & 6.1 \\
\hline & CareSens PRO & 78 & $46.5 \pm 3.4$ & 7.2 & $110.1 \pm 5.7$ & 5.2 & $376.7 \pm 48.3$ & 12.8 \\
\hline & Others & 69 & $38.2 \pm 2.6$ & 6.9 & $99.6 \pm 6.8$ & 6.8 & $282.4 \pm 23.0$ & 8.1 \\
\hline
\end{tabular}


Table 3. Continued

\begin{tabular}{|c|c|c|c|c|c|c|c|c|}
\hline \multirow[b]{2}{*}{ Manufacturer } & \multirow[b]{2}{*}{ Instrument } & \multirow[b]{2}{*}{ No. } & \multicolumn{2}{|c|}{ GGP-18-01 } & \multicolumn{2}{|c|}{ GGP-18-02 } & \multicolumn{2}{|c|}{ GGP-18-03 } \\
\hline & & & Mean \pm SD & $\begin{array}{l}\text { CV } \\
(\%)\end{array}$ & Mean $\pm S D$ & $\begin{array}{l}\text { CV } \\
(\%)\end{array}$ & Mean \pm SD & $\begin{array}{c}\text { CV } \\
(\%)\end{array}$ \\
\hline \multirow[t]{3}{*}{ Philosys } & Gmate check & & & & & & & \\
\hline & Gmate Origin & & & & & & & \\
\hline & Gmate WHeeL & & & & & & & \\
\hline \multirow[t]{4}{*}{ Roche } & Accu-Check Active & 198 & $78.3 \pm 2.3$ & 2.9 & $165.5 \pm 4.5$ & 2.7 & $419.2 \pm 11.1$ & 2.6 \\
\hline & Accu-Check Inform II & 411 & $68.5 \pm 1.5$ & 2.2 & $157.4 \pm 3.4$ & 2.2 & $406.9 \pm 9.5$ & 2.3 \\
\hline & Accu-Check Performa & 619 & $68.8 \pm 1.3$ & 2.0 & $158.4 \pm 3.4$ & 2.2 & $408.7 \pm 7.4$ & 1.8 \\
\hline & Others & & & & & & & \\
\hline \multirow[t]{11}{*}{ SD Biosensor } & SD CHECK GOLD (Meter 01GM10) & 36 & $63.7 \pm 1.7$ & 2.6 & $139.0 \pm 3.6$ & 2.6 & $375.4 \pm 9.1$ & 2.4 \\
\hline & SD CHECK GOLD (System 01GC12) & 7 & & & & & & \\
\hline & SD CodeFree (Meter 01GM11) & 117 & $55.6 \pm 5.3$ & 9.5 & $133.7 \pm 7.1$ & 5.3 & $353.9 \pm 11.3$ & 3.2 \\
\hline & SD CodeFree (System 01GC112) & 8 & & & & & & \\
\hline & SD GlucoNavii & & & & & & & \\
\hline & SD GlucoNavii GDH (Meter 01GM30) & & & & & & & \\
\hline & SD GlucoNavii GDH (System 01GC32) & & & & & & & \\
\hline & SD GlucoNavii Link 0.3 (Meter 01GM12) & 40 & $58.5 \pm 2.7$ & 4.7 & $145.0 \pm 4.9$ & 3.4 & $396.2 \pm 17.9$ & 4.5 \\
\hline & SD GlucoNavii Link 0.3 (System 01GC122) & 12 & $54.7 \pm 2.0$ & 3.6 & $130.8 \pm 3.2$ & 2.4 & $354.0 \pm 4.3$ & 1.2 \\
\hline & SD GlucoNavii NFC (Meter 01GM40) & 78 & $62.1 \pm 4.7$ & 7.6 & $136.1 \pm 11.7$ & 8.6 & $325.1 \pm 32.5$ & 10.0 \\
\hline & SD GlucoNavii NFC (System 01GC42) & 6 & & & & & & \\
\hline Wise Meditech & Wisecheck & & & & & & & \\
\hline Others & Others & 98 & $47.0 \pm 15.5$ & 33.1 & $106.5 \pm 17.1$ & 16.1 & $257.7 \pm 6.9$ & 2.7 \\
\hline
\end{tabular}

The instruments used were from the following companies: Abbott (Abbott Park, IL, USA), Allmedicus (Anyang, Korea), Arkray Inc. (St. Edina, MN, USA), Ascensia (Newbury, UK), GC Medis (Cheonan, Korea), Infopia (Anyang, Korea), i-SENS (Seoul, Korea), Philosys (Gunsan, Korea), Roche (Indianapolis, IN, USA), SD Biosensor (Suwon, Korea), and Wise Meditech (Anyang, Korea).

Abbreviation: SD, standard deviation; CV, coefficient of variance.

Glucometer는 전 세계적으로 가장 흔하게 사용되는 현장검사 장비로[3], 국내에서도 검사실이 있는 병원뿐만 아니라 검사실을 가지고 있지 않는 개인 의원이나 요양의료기관에서 광범위하게 사 용되고 있다. 그러나 이들 기관에서는 외부정도관리의 중요성을 알지 못하는 진단검사의학 관련 종사자가 아닌 경우가 대다수이 며, 따라서 이들의 glucose 현장검사에 대한 외부정도관리에 대 한 참여를 높이기 위해서는 외부정도관리의 장점과 필요성에 대 해 지속적으로 알리고 강조하는 것이 필요할 뿐만 아니라[7], 이들 의 참여를 확대할 수 있는 제도적인 장치의 마련도 필요할 것으로 생각한다. 노르웨이에서 시행되는 현장검사장비 외부정도관리프 로그램에는 요양원(nursing home)의 $96 \%$, 개인 의원의 $99 \%$ 가 point-of-care testing 외부정도관리에 참여하고 있으며, 이는 좋 은 본보기가 될 것이라고 생각한다[8].
측정장비의 수는 2018년 1차부터 2019년 2차까지 3,155, $3,262,3,788$, 그리고 3,803 개이며, 각 기관별로 평균 9.3 대, 9.7대, 10.6 대, 10.7 대가 회차별로 외부정도관리프로그램에 참 여한 것으로 나타나, 회차가 진행됨에 따라 참가기관의 평균 기 기의 대수가 늘어나는 것을 알 수 있다. 현재 한 기관당 최대 30 개까지의 glucometer의 참여가 가능하지만, 그 이상을 사용 하는 기관이 상당히 있을 것 판단하므로 기관당 참여 가능한 glucometer의 개수를 늘리는 것을 고려해볼 수 있으며, 이와 같 이 많은 수의 glucometer가 참가하는 기관의 경우 각 기관에서 나오는 결과에 대해 개별 결과뿐만 아니라 종합적인 결과를 제공 하는 것이 기관에 추가적인 정보를 제공하는 데 도움이 될 것으 로 판단한다.

Glucose 현장검사의 경우 검사실 간 정밀도의 차이가 제조 
Table 4. Distribution of instruments and mean, SD, and CVs (\%) for glucose point-of-care testing (GGP-18-04 to GGP-18-06)

\begin{tabular}{|c|c|c|c|c|c|c|c|c|}
\hline \multirow[b]{2}{*}{ Manufacturer } & \multirow[b]{2}{*}{ Instrument group } & \multirow[b]{2}{*}{ No. } & \multicolumn{2}{|c|}{ GGP-18-04 } & \multicolumn{2}{|c|}{ GGP-18-05 } & \multicolumn{2}{|c|}{ GGP-18-06 } \\
\hline & & & Mean $\pm S D$ & $\begin{array}{c}\text { CV } \\
(\%)\end{array}$ & Mean $\pm S D$ & $\begin{array}{l}\text { CV } \\
(\%)\end{array}$ & Mean $\pm S D$ & $\begin{array}{c}\text { CV } \\
\text { (\%) }\end{array}$ \\
\hline \multirow[t]{4}{*}{ Abbott } & Freestyle Optimum & 33 & $74.6 \pm 2.3$ & 3.1 & $151.4 \pm 6.5$ & 4.3 & $317.2 \pm 19.7$ & 6.2 \\
\hline & Freestyle Optimum Neo & 22 & $67.3 \pm 4.4$ & 6.6 & $164.8 \pm 12.4$ & 7.5 & $354.7 \pm 27.9$ & 7.9 \\
\hline & Freestyle Optimum $\mathrm{Neo} \mathrm{H}$ & 7 & & & & & & \\
\hline & Freestyle Optimum Xceed & 24 & $79.0 \pm 5.3$ & 6.7 & $161.1 \pm 6.0$ & 3.8 & $321.0 \pm 18.7$ & 5.8 \\
\hline \multirow[t]{4}{*}{ Allmedicus } & GlucoDr. Auto (AGM-4000) & 4 & & & & & & \\
\hline & GlucoDr. Plus (AGM-3000/B) & 68 & $46.3 \pm 3.2$ & 6.9 & $114.0 \pm 4.4$ & 3.9 & $264.5 \pm 11.5$ & 4.3 \\
\hline & GlucoDr. (AGM-2100) & 1 & & & & & & \\
\hline & Others & & & & & & & \\
\hline \multirow[t]{2}{*}{ Arkray Inc } & Glucocard 01 & 9 & & & & & & \\
\hline & Glucocard II & 2 & & & & & & \\
\hline \multirow[t]{3}{*}{ Ascensia } & Breeze 2 & & & & & & & \\
\hline & Contour plus & 43 & $55.4 \pm 2.1$ & 3.8 & $116.3 \pm 4.4$ & 3.7 & $270.3 \pm 7.3$ & 2.7 \\
\hline & Contour T & 23 & $41.3 \pm 13.7$ & 33.2 & $107.7 \pm 11.4$ & 10.6 & $321.1 \pm 44.1$ & 13.7 \\
\hline \multirow[t]{2}{*}{ GC Medis } & Green check & & & & & & & \\
\hline & Others & & & & & & & \\
\hline \multirow[t]{6}{*}{ Infopia } & GlucoLAB & 17 & $53.0 \pm 1.7$ & 3.2 & $117.9 \pm 2.0$ & 1.7 & $258.9 \pm 8.1$ & 2.8 \\
\hline & GlucoLAB Auto-coding & 20 & $52.4 \pm 7.0$ & 13.4 & $119.5 \pm 8.2$ & 6.9 & $296.9 \pm 14.4$ & 4.9 \\
\hline & Gluneo Lite & & & & & & & \\
\hline & Gluneo Plus & 133 & $57.5 \pm 3.4$ & 5.8 & $96.0 \pm 3.2$ & 3.4 & $236.9 \pm 6.5$ & 2.7 \\
\hline & Mirae 3.3G+ & 5 & & & & & & \\
\hline & Others & & & & & & & \\
\hline \multirow[t]{14}{*}{ i-SENS } & ACURA plus & & & & & & & \\
\hline & BAROZen & 66 & $40.1 \pm 2.9$ & 7.2 & $106.9 \pm 6.2$ & 5.8 & $284.2 \pm 25.7$ & 9.1 \\
\hline & BAROZen $\mathrm{H}$ & 114 & $39.9 \pm 2.1$ & 5.2 & $111.9 \pm 4.0$ & 3.6 & $315.0 \pm 12.2$ & 3.9 \\
\hline & BAROzen $\mathrm{H}$ expert & 421 & $55.8 \pm 3.8$ & 6.8 & $120.6 \pm 5.7$ & 4.8 & $326.6 \pm 14.9$ & 4.6 \\
\hline & BAROzen $\mathrm{H}$ expert plus & 83 & $54.5 \pm 3.6$ & 6.6 & $119.5 \pm 5.0$ & 4.2 & $326.6 \pm 13.8$ & 4.2 \\
\hline & BAROZen $\mathrm{H}$ plus & 145 & $54.2 \pm 3.6$ & 6.6 & $119.6 \pm 5.0$ & 4.1 & $327.2 \pm 16.5$ & 5.1 \\
\hline & BAROZen II & & & & & & & \\
\hline & BAROZen plus & & & & & & & \\
\hline & CareSens Dual & & & & & & & \\
\hline & CareSens II & 11 & $32.7 \pm 3.1$ & 9.6 & $98.2 \pm 3.8$ & 3.9 & $282.7 \pm 11.1$ & 3.9 \\
\hline & CareSens N & 83 & $41.4 \pm 3.9$ & 9.5 & $109.8 \pm 5.2$ & 4.7 & $302.1 \pm 20.0$ & 6.6 \\
\hline & CareSens N Voice & 118 & $38.9 \pm 3.3$ & 8.5 & $106.7 \pm 4.5$ & 4.2 & $302.1 \pm 16.3$ & 5.4 \\
\hline & CareSens PRO & 81 & $51.7 \pm 3.8$ & 7.3 & $119.8 \pm 5.2$ & 4.3 & $333.7 \pm 18.8$ & 5.6 \\
\hline & Others & 50 & $36.7 \pm 4.2$ & 11.3 & $107.2 \pm 4.4$ & 4.1 & $290.8 \pm 23.7$ & 8.2 \\
\hline
\end{tabular}


Table 4. Continued

\begin{tabular}{|c|c|c|c|c|c|c|c|c|}
\hline \multirow[b]{2}{*}{ Manufacturer } & \multirow[b]{2}{*}{ Instrument group } & \multirow[b]{2}{*}{ No. } & \multicolumn{2}{|c|}{ GGP-18-04 } & \multicolumn{2}{|c|}{ GGP-18-05 } & \multicolumn{2}{|c|}{ GGP-18-06 } \\
\hline & & & Mean $\pm S D$ & $\begin{array}{l}\text { CV } \\
(\%)\end{array}$ & Mean $\pm S D$ & $\begin{array}{l}\text { CV } \\
(\%)\end{array}$ & Mean $\pm S D$ & $\begin{array}{l}\text { CV } \\
(\%)\end{array}$ \\
\hline \multirow[t]{3}{*}{ Philosys } & Gmate check & & & & & & & \\
\hline & Gmate Origin & & & & & & & \\
\hline & Gmate WHeeL & & & & & & & \\
\hline \multirow[t]{4}{*}{ Roche } & Accu-Check Active & 178 & $77.9 \pm 2.7$ & 3.5 & $172.2 \pm 5.8$ & 3.4 & $428.1 \pm 9.4$ & 2.2 \\
\hline & Accu-Check Inform II & 413 & $69.5 \pm 1.4$ & 2.0 & $166.5 \pm 3.1$ & 1.9 & $403.6 \pm 8.3$ & 2.1 \\
\hline & Accu-Check Performa & 673 & $70.0 \pm 1.5$ & 2.1 & $166.2 \pm 3.4$ & 2.0 & $403.1 \pm 8.3$ & 2.1 \\
\hline & Others & & & & & & & \\
\hline \multirow[t]{11}{*}{ SD Biosensor } & SD CHECK GOLD (Meter 01GM10) & 37 & $60.2 \pm 3.8$ & 6.3 & $143.2 \pm 8.9$ & 6.2 & $372.1 \pm 17.3$ & 4.6 \\
\hline & SD CHECK GOLD (System 01GC12) & 6 & & & & & & \\
\hline & SD CodeFree (Meter 01GM11) & 87 & $58.1 \pm 5.7$ & 9.8 & $146.3 \pm 6.6$ & 4.5 & $368.1 \pm 11.8$ & 3.2 \\
\hline & SD CodeFree (System 01GC112) & 10 & $54.8 \pm 1.6$ & 2.9 & $137.3 \pm 2.5$ & 1.9 & $353.3 \pm 2.2$ & 0.6 \\
\hline & SD GlucoNavii & & & & & & & \\
\hline & SD GlucoNavii GDH (Meter 01GM30) & & & & & & & \\
\hline & SD GlucoNavii GDH (System 01GC32) & & & & & & & \\
\hline & SD GlucoNavii Link 0.3 (Meter 01GM12) & 40 & $58.6 \pm 4.6$ & 7.8 & $142.4 \pm 7.2$ & 5.1 & $373.2 \pm 13.1$ & 3.5 \\
\hline & SD GlucoNavii Link 0.3 (System 01GC122) & 12 & $57.8 \pm 4.8$ & 8.3 & $140.6 \pm 8.2$ & 5.8 & $337.6 \pm 14.4$ & 4.3 \\
\hline & SD GlucoNavii NFC (Meter 01GM40) & 82 & $64.1 \pm 3.6$ & 5.6 & $144.9 \pm 9.2$ & 6.3 & $348.8 \pm 33.7$ & 9.7 \\
\hline & SD GlucoNavii NFC (System 01GC42) & 11 & $61.5 \pm 1.3$ & 2.1 & $142.8 \pm 2.1$ & 1.5 & $349.3 \pm 7.7$ & 2.2 \\
\hline Wise Meditech & Wisecheck & & & & & & & \\
\hline Others & Others & 130 & $43.5 \pm 9.1$ & 20.8 & $113.2 \pm 12.3$ & 10.9 & $269.3 \pm 36.1$ & 12.2 \\
\hline
\end{tabular}

The instruments used were from the following companies: Abbott (Abbott Park, IL, USA), Allmedicus (Anyang, Korea), Arkray Inc. (St. Edina, MN, USA), Ascensia (Newbury, UK), GC Medis (Cheonan, Korea), Infopia (Anyang, Korea), i-SENS (Seoul, Korea), Philosys (Gunsan, Korea), Roche (Indianapolis, IN, USA), SD Biosensor (Suwon, Korea), and Wise Meditech (Anyang, Korea).

Abbreviation: SD, standard deviation; CV, coefficient of variance.

사별로 큰 것으로 나타났으며, 또한 같은 제조사 내에서도 장 비별로 차이를 나타냈다(Tables 3-6). 일반화학검사 프로그램 의 glucose 검사의 peer-group 변이계수(2.0\%-3.6\%)와 비교 하면, glucose 현장검사의 변이계수 평균이 더 높은 것으로 파 악된다[4]. 정밀도와 관련하여 glucometer가 $5 \%$ 의 변동성을 가질 경우 $8 \%-23 \%$ 의, 그리고 $10 \%$ 이상의 변이를 가질 때는 $16 \%-45 \%$ 의 인슐린 용량 조절에 차이를 나타낼 수 있음을 강조 하고 있다[9]. 외부정도관리결과를 통해 검사실 내의 정밀도는 파 악하기 어려우나 검사실 간 정밀도는 확인할 수 있으며, 이를 통해 일부 장비의 경우 $10 \%$ 를 넘는 경우를 자주 확인할 수 있었으며, 검사실 간의 비교 가능성에 대한 개선이 더 있어야 할 것으로 판단 된다.

현재 glucose 현장검사 외부정도관리프로그램에 사용되는
물질은 정도관리물질로 교환 가능성이 없으며, 이런 경우 일반 적으로 같은 시스템 내에서 교환 가능성만이 보장되기 때문에 장비 간의 계통오차를 비교하기 어렵다. 그러나 같은 장비 내에 서도 strip의 lot가 다를 경우 같은 측정시스템 내에서 정도관 리물질에 대한 교환 가능성이 없는 것이 알려졌으며[10], 그럴 경우 해당 장비에 대한 검사실 간 변이계수가 높게 나타날 수 있다.

지난 2년간 총 4회의 외부정도관리프로그램을 시행하는 동안 특정 장비의 한 회차에서 검사실 간 변이계수가 이전 회차들과 매 우 큰 차이를 보이는 것을 확인할 수 있었고(Fig. 2), 이는 참가기 관의 문제이기보다는 strip lot 간 변이 가능성이 가장 예상된다. 한편, 정도관리물질에 대한 lot 간 변이가 실제 환자의 결과에 영 향을 미치는 가능성이 있는 만큼[10,11], 제조사는 lot 간 변이가 
Table 5. Distribution of instruments and mean, SD, and CVs (\%) for glucose point-of-care testing (GGP-19-01 to GGP-19-03)

\begin{tabular}{|c|c|c|c|c|c|c|c|c|}
\hline \multirow[b]{2}{*}{ Manufacturer } & \multirow[b]{2}{*}{ Instrument group } & \multirow[b]{2}{*}{ No. } & \multicolumn{2}{|c|}{ GGP-19-01 } & \multicolumn{2}{|c|}{ GGP-19-02 } & \multicolumn{2}{|c|}{ GGP-19-03 } \\
\hline & & & Mean \pm SD & $\begin{array}{c}\text { CV } \\
(\%)\end{array}$ & Mean $\pm S D$ & $\begin{array}{c}\text { CV } \\
(\%)\end{array}$ & Mean $\pm S D$ & $\begin{array}{c}\text { CV } \\
(\%)\end{array}$ \\
\hline \multirow[t]{4}{*}{ Abbott } & Freestyle Optimum & 38 & $72.8 \pm 2.4$ & 3.3 & $146.3 \pm 2.8$ & 1.9 & $309.6 \pm 11.5$ & 3.7 \\
\hline & Freestyle Optimum Neo & 33 & $62.9 \pm 5.9$ & 8.9 & $146.9 \pm 8.2$ & 5.6 & $309.8 \pm 15.0$ & 4.9 \\
\hline & Freestyle Optimum $\mathrm{Neo} \mathrm{H}$ & 8 & & & & & & \\
\hline & Freestyle Optimum Xceed & 19 & $71.8 \pm 2.9$ & 4.1 & $152.2 \pm 4.1$ & 2.7 & $306.2 \pm 13.0$ & 4.2 \\
\hline \multirow[t]{4}{*}{ Allmedicus } & GlucoDr. Auto (AGM-4000) & 10 & $32.6 \pm 3.8$ & 11.7 & $96.9 \pm 8.0$ & 8.2 & $276.0 \pm 14.9$ & 5.4 \\
\hline & GlucoDr. Plus (AGM-3000/B) & 26 & $41.2 \pm 3.0$ & 7.4 & $109.4 \pm 6.7$ & 6.1 & $260.5 \pm 10.6$ & 4.1 \\
\hline & GlucoDr. (AGM-2100) & 1 & & & & & & \\
\hline & Others & 30 & $36.6 \pm 2.2$ & 5.9 & $98.0 \pm 4.8$ & 4.9 & $278.9 \pm 16.8$ & 6.0 \\
\hline \multirow[t]{2}{*}{ Arkray Inc. } & Glucocard 01 & 10 & $37.1 \pm 0.9$ & 2.4 & $94.3 \pm 1.4$ & 1.5 & $281.5 \pm 2.5$ & 0.9 \\
\hline & Glucocard II & 1 & & & & & & \\
\hline \multirow[t]{3}{*}{ Ascensia } & Breeze 2 & & & & & & & \\
\hline & Contour plus & 58 & $52.8 \pm 1.9$ & 3.6 & $115.6 \pm 3.2$ & 2.8 & $274.4 \pm 9.2$ & 3.4 \\
\hline & Contour T & 14 & $49.0 \pm 7.1$ & 14.5 & $102.7 \pm 20.1$ & 19.5 & $285.9 \pm 34.7$ & 12.1 \\
\hline \multirow[t]{2}{*}{ GC Medis } & Green check & & & & & & & \\
\hline & Others & & & & & & & \\
\hline \multirow[t]{6}{*}{ Infopia } & GlucoLAB & 20 & $51.0 \pm 2.1$ & 4.1 & $122.0 \pm 4.1$ & 3.3 & $314.2 \pm 12.4$ & 3.9 \\
\hline & GlucoLAB Auto-coding & 23 & $51.5 \pm 3.1$ & 6.1 & $119.3 \pm 3.5$ & 3.0 & $301.8 \pm 7.5$ & 2.5 \\
\hline & Gluneo Lite & & & & & & & \\
\hline & Gluneo Plus & 165 & $51.2 \pm 4.1$ & 7.9 & $94.5 \pm 7.2$ & 7.6 & $237.0 \pm 7.5$ & 3.2 \\
\hline & Mirae 3.3G+ & 5 & & & & & & \\
\hline & Others & & & & & & & \\
\hline \multirow[t]{14}{*}{ i-SENS } & ACURA plus & 48 & $40.5 \pm 3.5$ & 8.7 & $111.5 \pm 5.9$ & 5.3 & $301.2 \pm 17.7$ & 5.9 \\
\hline & BAROZen & 59 & $39.8 \pm 3.7$ & 9.2 & $107.2 \pm 7.4$ & 6.9 & $288.0 \pm 21.8$ & 7.6 \\
\hline & BAROZen $\mathrm{H}$ & 93 & $39.2 \pm 1.4$ & 3.7 & $113.2 \pm 3.4$ & 3.0 & $306.7 \pm 3.2$ & 1.0 \\
\hline & BAROzen $\mathrm{H}$ expert & 442 & $51.7 \pm 2.7$ & 5.2 & $113.3 \pm 6.2$ & 5.5 & $319.6 \pm 12.3$ & 3.8 \\
\hline & BAROzen $\mathrm{H}$ expert plus & 203 & $51.6 \pm 2.2$ & 4.2 & $113.1 \pm 3.3$ & 2.9 & $318.3 \pm 7.6$ & 2.4 \\
\hline & BAROZen $\mathrm{H}$ plus & 170 & $50.3 \pm 3.1$ & 6.8 & $110.9 \pm 10.3$ & 9.3 & $314.6 \pm 43.8$ & 13.9 \\
\hline & BAROZen II & 10 & $39.0 \pm 0.9$ & 2.2 & $105.8 \pm 0.7$ & 0.6 & $298.8 \pm 7.3$ & 2.4 \\
\hline & BAROZen plus & 67 & $48.4 \pm 2.5$ & 5.2 & $99.1 \pm 2.6$ & 2.6 & $267.1 \pm 6.2$ & 6.3 \\
\hline & CareSens Dual & 10 & & & $114.1 \pm 2.9$ & 2.5 & $416.2 \pm 10.7$ & 2.6 \\
\hline & CareSens II & 11 & $32.5 \pm 3.8$ & 11.7 & $98.6 \pm 6.8$ & 6.9 & $284.0 \pm 43.0$ & 15.1 \\
\hline & CareSens N & 61 & $42.6 \pm 3.8$ & 8.8 & $114.4 \pm 7.4$ & 6.5 & $318.2 \pm 14.1$ & 4.4 \\
\hline & CareSens N Voice & 113 & $35.3 \pm 2.1$ & 5.9 & $107.1 \pm 5.7$ & 5.3 & $300.6 \pm 19.4$ & 6.4 \\
\hline & CareSens PRO & 107 & $49.3 \pm 3.4$ & 6.9 & $114.9 \pm 5.0$ & 4.4 & $370.3 \pm 33.5$ & 9.1 \\
\hline & others & & & & & & & \\
\hline
\end{tabular}


Table 5. Continued

\begin{tabular}{|c|c|c|c|c|c|c|c|c|}
\hline \multirow[b]{2}{*}{ Manufacturer } & \multirow[b]{2}{*}{ Instrument group } & \multirow[b]{2}{*}{ No. } & \multicolumn{2}{|c|}{ GGP-19-01 } & \multicolumn{2}{|c|}{ GGP-19-02 } & \multicolumn{2}{|c|}{ GGP-19-03 } \\
\hline & & & Mean \pm SD & $\begin{array}{c}\text { CV } \\
(\%)\end{array}$ & Mean $\pm S D$ & $\begin{array}{l}\text { CV } \\
(\%)\end{array}$ & Mean \pm SD & $\begin{array}{l}\text { CV } \\
(\%)\end{array}$ \\
\hline \multirow[t]{3}{*}{ Philosys } & Gmate check & 46 & $48.7 \pm 3.7$ & 7.7 & $121.8 \pm 4.3$ & 3.5 & $332.9 \pm 22.3$ & 6.7 \\
\hline & Gmate Origin & 28 & $61.6 \pm 3.4$ & 5.5 & $135.4 \pm 3.9$ & 2.9 & $340.0 \pm 6.8$ & 2.0 \\
\hline & Gmate WHeeL & 11 & $55.4 \pm 1.3$ & 2.3 & $124.4 \pm 1.6$ & 1.3 & $324.5 \pm 1.8$ & 0.5 \\
\hline \multirow[t]{4}{*}{ Roche } & Accu-Check Active & 186 & $76.3 \pm 1.8$ & 2.3 & $170.5 \pm 4.3$ & 2.5 & $434.3 \pm 12.2$ & 2.8 \\
\hline & Accu-Check Inform II & 485 & $67.2 \pm 1.4$ & 2.1 & $161.6 \pm 3.0$ & 1.9 & $103.2 \pm 7.1$ & 1.8 \\
\hline & Accu-Check Performa & 722 & $66.5 \pm 1.8$ & 2.8 & $159.5 \pm 3.4$ & 2.1 & $398.8 \pm 7.6$ & 1.9 \\
\hline & Others & & & & & & & \\
\hline \multirow[t]{11}{*}{ SD Biosensor } & SD CHECK GOLD (Meter 01GM10) & 40 & $63.8 \pm 2.1$ & 3.3 & $145.7 \pm 6.1$ & 4.1 & $374.2 \pm 9.9$ & 2.6 \\
\hline & SD CHECK GOLD (System 01GC12) & & & & & & & \\
\hline & SD CodeFree (Meter 01GM11) & 123 & $62.4 \pm 5.3$ & 8.4 & $146.8 \pm 7.8$ & 5.3 & $360.6 \pm 15.0$ & 4.2 \\
\hline & SD CodeFree (System 01GC112) & 8 & & & & & & \\
\hline & SD GlucoNavii & 30 & $59.6 \pm 2.8$ & 4.8 & $134.0 \pm 6.9$ & 5.2 & $339.2 \pm 21.0$ & 6.5 \\
\hline & SD GlucoNavii GDH (Meter 01GM30) & 14 & $59.4 \pm 4.7$ & 8.0 & $128.1 \pm 5.0$ & 3.9 & $301.5 \pm 13.0$ & 4.3 \\
\hline & SD GlucoNavii GDH (System 01GC32) & 6 & & & & & & \\
\hline & SD GlucoNavii Link 0.3 (Meter 01GM12) & 41 & $60.2 \pm 3.1$ & 5.2 & $145.2 \pm 6.4$ & 4.4 & $385.7 \pm 17.2$ & 4.5 \\
\hline & SD GlucoNavii Link 0.3 (System 01GC122) & & & & & & & \\
\hline & SD GlucoNavii NFC (Meter 01GM40) & 75 & $60.4 \pm 4.2$ & 6.9 & $137.1 \pm 8.4$ & 6.1 & $332.2 \pm 29.9$ & 9.0 \\
\hline & SD GlucoNavii NFC (System 01GC42) & & & & & & & \\
\hline Wise Meditech & Wisecheck & 13 & $38.8 \pm 0.8$ & 1.9 & $112.7 \pm 1.8$ & 1.6 & $337.6 \pm 5.3$ & 1.6 \\
\hline Others & Others & 108 & $39.1 \pm 11.2$ & 28.5 & $113.2 \pm 13.8$ & 12.2 & $294.6 \pm 39.1$ & 13.3 \\
\hline
\end{tabular}

The instruments used were from the following companies: Abbott (Abbott Park, IL, USA), Allmedicus (Anyang, Korea), Arkray Inc. (St. Edina, MN, USA), Ascensia (Newbury, UK), GC Medis (Cheonan, Korea), Infopia (Anyang, Korea), i-SENS (Seoul, Korea), Philosys (Gunsan, Korea), Roche (Indianapolis, IN, USA), SD Biosensor (Suwon, Korea), and Wise Meditech (Anyang, Korea).

Abbreviation: SD, standard deviation; CV, coefficient of variance.

의심되는 경우 이것이 실제 검체에 영향을 미치는 지에 대한 확인 과정이 필요하다. 또한 외부정도관리프로그램 운영 시 strip lot 번호에 대한 정보를 함께 얻는 것이 필요하며, 이를 통해 lot 간의 변이를 추적할 수 있으며, lot별 참값을 기관에 제공하는 것을 통 해 실제 문제가 없는 기관들이 평가를 잘못 받는 것을 막을 수 있 다[11].
결론적으로, 2018년부터 2019년까지 glucose 현장검사에 참가 한 기관 수 및 장비 수는 이전에 비해 지속적으로 증가하고 있으며, glucose 현장검사장비의 변이계수는 중앙검사실의 값보다 낮은 것 으로 확인되었다. 검사의 질 향상을 위해 더 많은 기관들이 참여할 수 있도록 지속적인 노력을 기울일 필요가 있으며, strip lot 간 변이 를 확인하기 위해 이에 대한 정보를 추가적으로 얻는 것이 필요하다. 
Table 6. Distribution of instruments and mean, SD, and CVs (\%) for glucose point-of-care testing (GGP-19-04 to GGP-19-06)

\begin{tabular}{|c|c|c|c|c|c|c|c|c|}
\hline \multirow[b]{2}{*}{ Manufacturer } & \multirow[b]{2}{*}{ Instrument group } & \multirow[b]{2}{*}{ No. } & \multicolumn{2}{|c|}{ GGP-19-04 } & \multicolumn{2}{|c|}{ GGP-19-05 } & \multicolumn{2}{|c|}{ GGP-19-06 } \\
\hline & & & Mean $\pm S D$ & $\begin{array}{l}C V \\
(\%)\end{array}$ & Mean \pm SD & $\begin{array}{l}\mathrm{CV} \\
(\%)\end{array}$ & Mean \pm SD & $\begin{array}{c}\text { CV } \\
(\%)\end{array}$ \\
\hline \multirow[t]{4}{*}{ Abbott } & Freestyle Optimum & 22 & $157.9 \pm 6.8$ & 4.3 & $321.7 \pm 9.9$ & 3.1 & $82.7 \pm 4.3$ & 5.2 \\
\hline & Freestyle Optimum Neo & 31 & $156.3 \pm 13.8$ & 8.8 & $329.3 \pm 31.8$ & 9.6 & $73.0 \pm 4.7$ & 6.4 \\
\hline & Freestyle Optimum $\mathrm{Neo} \mathrm{H}$ & 8 & & & & & & \\
\hline & Freestyle Optimum Xceed & 19 & $153.6 \pm 5.2$ & 3.4 & $296.1 \pm 20.6$ & 7.0 & $77.3 \pm 4.2$ & 5.5 \\
\hline \multirow[t]{4}{*}{ Allmedicus } & GlucoDr. Auto (AGM-4000) & 10 & $102.1 \pm 4.7$ & 4.6 & & & $37.9 \pm 4.4$ & 11.7 \\
\hline & GlucoDr. Plus (AGM-3000/B) & 15 & $116.4 \pm 6.4$ & 5.5 & $315.5 \pm 43.0$ & 13.6 & $50.5 \pm 16.3$ & 32.4 \\
\hline & GlucoDr. (AGM-2100) & & & & & & & \\
\hline & Others & 41 & $105.4 \pm 5.0$ & 4.8 & $270.1 \pm 22.4$ & 8.3 & $41.5 \pm 2.8$ & 6.6 \\
\hline \multirow[t]{2}{*}{ Arkray Inc. } & Glucocard 01 & 10 & $86.4 \pm 2.3$ & 2.6 & $266.4 \pm 4.6$ & 1.7 & $37.5 \pm 1.0$ & 2.6 \\
\hline & Glucocard II & 1 & & & & & & \\
\hline \multirow[t]{3}{*}{ Ascensia } & Breeze 2 & & & & & & & \\
\hline & Contour plus & 62 & $117.8 \pm 3.2$ & 2.7 & $272.0 \pm 7.7$ & 2.8 & $59.0 \pm 1.5$ & 2.5 \\
\hline & Contour T & & & & & & & \\
\hline \multirow[t]{2}{*}{ GC Medis } & Green check & 26 & $108.5 \pm 5.6$ & 5.2 & $254.0 \pm 5.8$ & 2.3 & $33.8 \pm 2.3$ & 6.7 \\
\hline & Others & 4 & & & & & & \\
\hline \multirow[t]{6}{*}{ Infopia } & Gluneo Lite & & & & & & & \\
\hline & Gluneo Plus & 190 & $109.7 \pm 10.4$ & 9.5 & $258.3 \pm 22.7$ & 8.8 & $64.4 \pm 6.3$ & 9.8 \\
\hline & GlucoLAB Auto-coding & 22 & $108.8 \pm 2.4$ & 2.2 & $257.5 \pm 18.8$ & 7.3 & $62.0 \pm 1.6$ & 2.6 \\
\hline & Mirae 3.3G+ & 5 & & & & & & \\
\hline & GlucoLAB & & & & & & & \\
\hline & Others & & & & & & & \\
\hline \multirow[t]{14}{*}{ i-SENS } & ACURA plus & 52 & $107.1 \pm 6.4$ & 6.0 & $282.7 \pm 23.6$ & 8.3 & $43.0 \pm 4.5$ & 10.5 \\
\hline & BAROZen & 61 & $104.3 \pm 9.3$ & 9.0 & $239.0 \pm 44.1$ & 18.4 & $42.9 \pm 3.1$ & 7.2 \\
\hline & BAROZen $\mathrm{H}$ & 59 & $103.1 \pm 5.2$ & 2.0 & $293.1 \pm 11.4$ & 3.9 & $41.4 \pm 2.9$ & 6.9 \\
\hline & BAROzen $\mathrm{H}$ expert & 396 & $136.6 \pm 10.1$ & 7.4 & $395.7 \pm 27.8$ & 7.0 & $54.4 \pm 2.8$ & 5.2 \\
\hline & BAROzen $\mathrm{H}$ expert plus & 321 & $136.2 \pm 11.1$ & 8.2 & $388.8 \pm 25.7$ & 6.6 & $54.6 \pm 2.5$ & 4.5 \\
\hline & BAROZen $\mathrm{H}$ plus & 154 & $129.6 \pm 19.4$ & 15.0 & $379.5 \pm 69.4$ & 18.3 & $53.7 \pm 3.6$ & 6.7 \\
\hline & BAROZen II & 14 & $108.2 \pm 2.0$ & 1.9 & $287.9 \pm 10.3$ & 3.6 & $44.6 \pm 1.9$ & 4.2 \\
\hline & BAROZen plus & 134 & $109.7 \pm 6.7$ & 6.1 & $268.4 \pm 8.8$ & 3.3 & $55.9 \pm 3.0$ & 5.3 \\
\hline & CareSens Dual & 10 & $59.9 \pm 2.8$ & 4.7 & $136.8 \pm 4.4$ & 3.2 & $400.1 \pm 4.8$ & 1.2 \\
\hline & CareSens II & 1 & & & & & & \\
\hline & CareSens N & 60 & $106.2 \pm 6.1$ & 5.7 & $298.2 \pm 18.1$ & 6.1 & $44.5 \pm 2.0$ & 4.5 \\
\hline & CareSens N Voice & 97 & $105.7 \pm 4.2$ & 3.9 & $292.2 \pm 13.3$ & 4.5 & $43.6 \pm 2.9$ & 6.6 \\
\hline & CareSens PRO & 104 & $138.3 \pm 6.9$ & 5.0 & $431.5 \pm 30.0$ & 6.9 & $54.1 \pm 3.3$ & 6.1 \\
\hline & others & & & & & & & \\
\hline
\end{tabular}


Table 6. Continued

\begin{tabular}{|c|c|c|c|c|c|c|c|c|}
\hline \multirow[b]{2}{*}{ Manufacturer } & \multirow[b]{2}{*}{ Instrument group } & \multirow[b]{2}{*}{ No. } & \multicolumn{2}{|c|}{ GGP-19-04 } & \multicolumn{2}{|c|}{ GGP-19-05 } & \multicolumn{2}{|c|}{ GGP-19-06 } \\
\hline & & & Mean \pm SD & $\begin{array}{c}\text { CV } \\
(\%)\end{array}$ & Mean \pm SD & $\begin{array}{l}\text { CV } \\
(\%)\end{array}$ & Mean $\pm S D$ & $\begin{array}{l}\text { CV } \\
(\%)\end{array}$ \\
\hline \multirow[t]{3}{*}{ Philosys } & Gmate check & 46 & $121.0 \pm 12.6$ & 10.4 & $328.9 \pm 34.0$ & 10.3 & $50.4 \pm 5.2$ & 10.3 \\
\hline & Gmate Origin & 26 & $112.7 \pm 3.7$ & 3.3 & $315.9 \pm 6.0$ & 1.9 & $46.8 \pm 1.9$ & 4.2 \\
\hline & Gmate WHeeL & 11 & $101.5 \pm 1.0$ & 1.0 & $284.6 \pm 1.9$ & 0.7 & $44.0 \pm 0.9$ & 2.0 \\
\hline \multirow[t]{4}{*}{ Roche } & Accu-Check Active & 153 & $189.3 \pm 13.6$ & 7.2 & $447.2 \pm 20.2$ & 4.5 & $93.7 \pm 8.9$ & 9.5 \\
\hline & Accu-Check Inform II & 461 & $185.9 \pm 21.8$ & 11.7 & $441.4 \pm 62.0$ & 14.1 & $76.6 \pm 4.5$ & 5.9 \\
\hline & Accu-Check Performa & 722 & $169.3 \pm 7.5$ & 4.0 & $405.8 \pm 10.9$ & 2.7 & $75.1 \pm 4.6$ & 6.2 \\
\hline & Others & 14 & $999.0 \pm 0.0$ & 0.0 & $999.0 \pm 0.0$ & 0.0 & $999.0 \pm 0.0$ & 0.0 \\
\hline \multirow[t]{11}{*}{ SD Biosensor } & SD CHECK GOLD (Meter 01GM10) & 38 & $103.6 \pm 39.3$ & 37.9 & $249.0 \pm 119.6$ & 48.0 & $210.3 \pm 143.0$ & 68.0 \\
\hline & SD CHECK GOLD (System 01GC12) & & & & & & & \\
\hline & SD CodeFree (Meter 01GM11) & 134 & $146.0 \pm 7.1$ & 4.9 & $364.5 \pm 14.3$ & 39 & $69.9 \pm 5.1$ & 7.4 \\
\hline & SD CodeFree (System 01GC112) & 8 & & & & & & \\
\hline & SD GlucoNavii & & & & & & & \\
\hline & SD GlucoNavii GDH (Meter 01GM30) & 49 & $62.4 \pm 7.1$ & 11.4 & $124.4 \pm 12.6$ & 10.1 & $292.0 \pm 17.2$ & 5.9 \\
\hline & SD GlucoNavii GDH (System 01GC32) & 19 & $147.1 \pm 5.5$ & 3.7 & $346.2 \pm 23.2$ & 6.7 & $63.8 \pm 4.0$ & 6.3 \\
\hline & SD GlucoNavii Link 0.3 (Meter 01GM12) & 41 & $133.6 \pm 8.5$ & 6.4 & $215.6 \pm 113.7$ & 52.7 & $36.6 \pm 22.7$ & 621 \\
\hline & SD GlucoNavii Link 0.3 (System 01GC122) & & & & & & & \\
\hline & SD GlucoNavii NFC (Meter 01GM40) & 65 & $1,413 \pm 52$ & 3.7 & $338.9 \pm 27.6$ & 8.1 & $66.6 \pm 8.2$ & 12.3 \\
\hline & SD GlucoNavii NFC (System 01GC42) & & & & & & & \\
\hline Wise Meditech & Wisecheck & 13 & $119.5 \pm 0.9$ & 0.8 & $335.7 \pm 4.0$ & 1.2 & $44.4 \pm 0.5$ & 1.1 \\
\hline Others & Others & 74 & $90.7 \pm 22.9$ & 25.3 & $226.5 \pm 58.0$ & 25.6 & $37.7 \pm 6.9$ & 18.2 \\
\hline
\end{tabular}

The instruments used were from the following companies: Abbott (Abbott Park, IL, USA), Allmedicus (Anyang, Korea), Arkray Inc. (St. Edina, MN, USA), Ascensia (Newbury, UK), GC Medis (Cheonan, Korea), Infopia (Anyang, Korea), i-SENS (Seoul, Korea), Philosys (Gunsan, Korea), Roche (Indianapolis, IN, USA), SD Biosensor (Suwon, Korea), and Wise Meditech (Anyang, Korea).

Abbreviation: SD, standard deviation; CV, coefficient of variance.

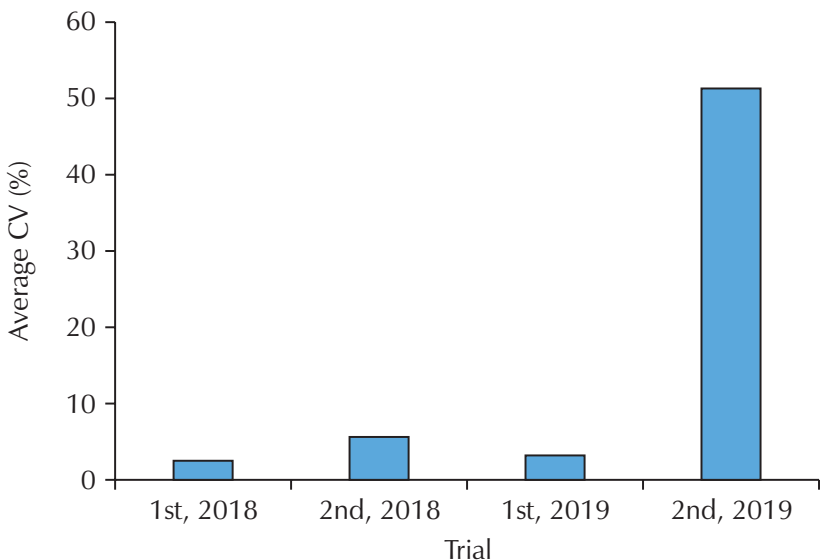

Fig. 2. An example of abrupt increase in coefficient of variance (CV) for 2nd 2019 trial of certain instrument.

\section{ORCID}

Jinsook Lim

Gye Cheol Kwon

https://orcid.org/0000-0003-2814-3284

https://orcid.org/0000-0002-4886-0590 


\section{References}

1. Bietenbeck A, Geilenkeuser WJ, Klawonn F, Spannagl M, Nauck M, Petersmann A, et al. External quality assessment schemes for glucose measurements in Germany: factors for successful participation, analytical performance and medical impact. Clin Chem Lab Med 2018;56:1238-50.

2. Clarke SF, Foster JR. A history of blood glucose meters and their role in self-monitoring of diabetes mellitus. $\mathrm{Br} \mathrm{J}$ Biomed Sci 2012;69:83-93.

3. Tonyushkina K, Nichols JH. Glucose meters: a review of technical challenges to obtaining accurate results. J Diabetes Sci Technol 2009;3:971-80.

4. Lee YW. Annual report of the Korean Association of External Quality Assessment Service on general chemistry (2019). Lab Med Qual Assur 2020;42:97-111.

5. Bukve T, Stavelin A, Sandberg S. Effect of participating in a quality improvement system over time for point-of-care c-reactive protein, glucose, and hemoglobin testing. Clin Chem 2016;62:1474-81.

6. Bukve T, Roraas T, Riksheim BO, Christensen NG, Sandberg S. Point-of-care urine albumin in general practice offices: effect of participation in an external quality assurance scheme. Clin Chem Lab Med 2015;53:45-51.

7. Stavelin A, Sandberg S. Essential aspects of external quality assurance for point-of-care testing. Biochem Med (Zagreb) 2017;27:81-5.

8. Stavelin A, Sandberg S. Harmonization activities of Noklus: a quality improvement organization for point-of-care laboratory examinations. Clin Chem Lab Med 2018;57:106-14.

9. Boyd JC, Bruns DE. Quality specifications for glucose meters: assessment by simulation modeling of errors in insulin dose. Clin Chem 2001;47:209-14.

10. Stavelin A, Riksheim BO, Christensen NG, Sandberg S. The importance of reagent lot registration in external quality assurance/proficiency testing schemes. Clin Chem 2016;62:708-15.

11. Kristensen GB, Christensen NG, Thue G, Sandberg S. Between-lot variation in external quality assessment of glucose: clinical importance and effect on participant performance evaluation. Clin Chem 2005;51:1632-6. 